\title{
Approaches to Scale Development in Chinese Management Research
}

\section{Jiing-Lih Farh, Albert A. Cannella, Jr ${ }^{1}$ and Cynthia Lee ${ }^{2}$}

The Hong Kong University of Science and Technology, China, ${ }^{1}$ Arizona State University, USA, and ${ }^{2}$ Northeastern University, USA

ARSTRAct We classify survey scales or measures currently used in Chinese management research along two dimensions - the source of the scale and expectations about its cultural specificity. Based on these two dimensions, we differentiate four approaches to scale development: translation, adaptation, de-contextualization, and contextualization. We describe the key assumptions, strengths and limitations of each approach and their roles in Chinese management research. We illustrate the four approaches by commenting on the five articles in this special issue.

KEYWORDS scale development, Chinese management contexts, translation, adaptation, contextualization, de-contextualization

The mechanic, who wishes to do his work well, must first sharpen his tools.

Confucius

\section{INTRODUGTION}

Some 2500 years ago, Confucius admonished that one must have good tools to do good work. When it comes to conducting high quality empirical research, it is imperative that we have valid measuring instruments. As Korman (1974, p. 194) has said aptly, "The point is not that adequate measurement is "nice". It is necessary, crucial, etc. Without it we have nothing'.

Although this is a received doctrine in the behavioural sciences, it has not always been heeded in practice. In the history of organizational science, there are many examples of researchers who rushed to undertake substantive research without first crafting valid research instruments (for an overview of this problem, see Schwab, 1980). The drawbacks of such an approach are many (DeVellis, 2003). First, research findings based on invalid instruments are compromised and sometimes seriously flawed. Secondly, both precious research resources and time are wasted. Thirdly, knowledge development in the area is hampered. 
In Chinese contexts, where empirical organizational research is nascent, the need for theory-based valid research instruments takes on even greater urgency. First, there is a paucity of measures with demonstrated validity for the local context. Secondly, most measures in use are Chinese translations of Western measures. Questions may be raised about the suitability of these translated or adapted scales for accurately capturing constructs in Chinese contexts. These concerns go well beyond language differences and the quality of translation procedures. Cultural factors such as a divergent construal of selves, the individualismcollectivism dynamic, and power distance, as well as institutional factors such as capital markets, third-party contract enforcement (legal systems), the businessgovernment interface, and the professionalization of management can all have an impact on organizational research, and make instruments originally developed for Western settings unsuitable for organizational research in China.

As Tsui $(2006$, p. 9) has aptly pointed out, 'To further Chinese management research and develop valid knowledge, contextualization in measurement is not only desirable, but essential'. In this introductory essay to the special issue on 'Developing Valid Measures for Chinese Management Research', we discuss four major approaches to scale development in Chinese management research. Although we contextualize our discussion using China as the focus, we expect these approaches to also be relevant for similar applications in other contexts. We then show how the five papers in this special issue, all of which were accepted after a rigorous development review process and several rounds of revisions by the authors, are good illustrations of at least three of the four approaches. We conclude by offering some action-oriented suggestions for those who wish to conduct research in new contexts with the objective of contributing to the stock of global management knowledge.

\section{FOUR SCALE DEVELOPMENT APPROAGHES}

Broadly speaking, survey scales ${ }^{[1]}$ or measures currently used in Chinese management research can be classified along two dimensions - the source of the scale, and expectations about its cultural specificity. The source of the scale indicates whether the scale is developed from scratch or derived from an existing measure. Expectations about cultural specificity involve the researcher's expectations about whether the new scale is universal ('etic') or specific to a cultural context ('emic'). Juxtaposing these two dimensions generates four approaches to scale development. The first approach - translation - involves using a direct translation of an existing Western scale (the source language scale) to create a Chinese language version of the scale (the target language scale). The second approach - adaptation - also involves translating an existing scale, but with some modification to create a more meaningful Chinese version. A third approach - de-contextualization - involves assembling a scale from scratch in a Chinese context, under the assumption that 
Table 1. Four types of scale development approaches in Chinese management research

\begin{tabular}{llr}
\hline \hline Source of the scale & \multicolumn{2}{c}{ Expectations about cultural specificity } \\
\cline { 2 - 3 } & \multicolumn{1}{c}{ Etic orientation } & Emic orientation \\
\hline Use or modify an existing scale & $\begin{array}{l}\text { Translation } \\
\text { Develop a new scale }\end{array}$ & $\begin{array}{l}\text { Adaptation } \\
\text { Contextualization }\end{array}$ \\
\hline \hline
\end{tabular}

the target construct is etic (i.e., universal or culturally invariant). Finally the fourth approach - contextualization - involves developing a scale from scratch in a Chinese context, but under the assumption that the target construct is emic (i.e., relevant to China only). The classification of the four approaches is in Table 1.

Each approach listed in Table 1 has unique strengths and weaknesses. Each can play a significant role in supplying tools to researchers conducting research in Chinese contexts. Below, we describe the characteristics of the four approaches and their roles in contributing to Chinese management research. Table 2 lists the key assumptions, strengths and limitations associated with each approach.

\section{The Translation Approach}

The term 'translation' is used broadly in the cross-cultural literature and can mean literal or simple translation, adapting parts of the instrument or assembling an entirely new instrument (Van de Vijver and Leung, 1997). In this essay, our usage is restricted to literal or simple translation. The translation approach consists of borrowing from an existing Western scale (also called a source language scale) for a target construct and then literally translating it into a Chinese version (also called a target language scale) for research in Chinese contexts. The translation approach is grounded on two major assumptions: (i) the meaning of the target construct is equivalent across cultures; and (ii) a high quality unbiased source language scale is available for the target construct. Construct equivalence (also called conceptual equivalence) has been used varyingly in the literature. For example, Behling and Law (2000, p. 16) defined it as the degree to which a concept, independent of the words used to operationalize it, exists in the same form in the source and target cultures. Hambleton and Patsula (1998, p. 159) refer to it as the extent to which a test measures the same construct in each language version. Van de Vijver and Leung $(1997$, p. 8) equate it with structural equivalence, meaning that an instrument measures the same construct in two cultures. Following Behling and Law (2000), we define construct equivalence to mean that the construct definition, content domain and the empirical representations of the content domain are the same across cultures. Under these assumptions, the goal of literal translation is to 


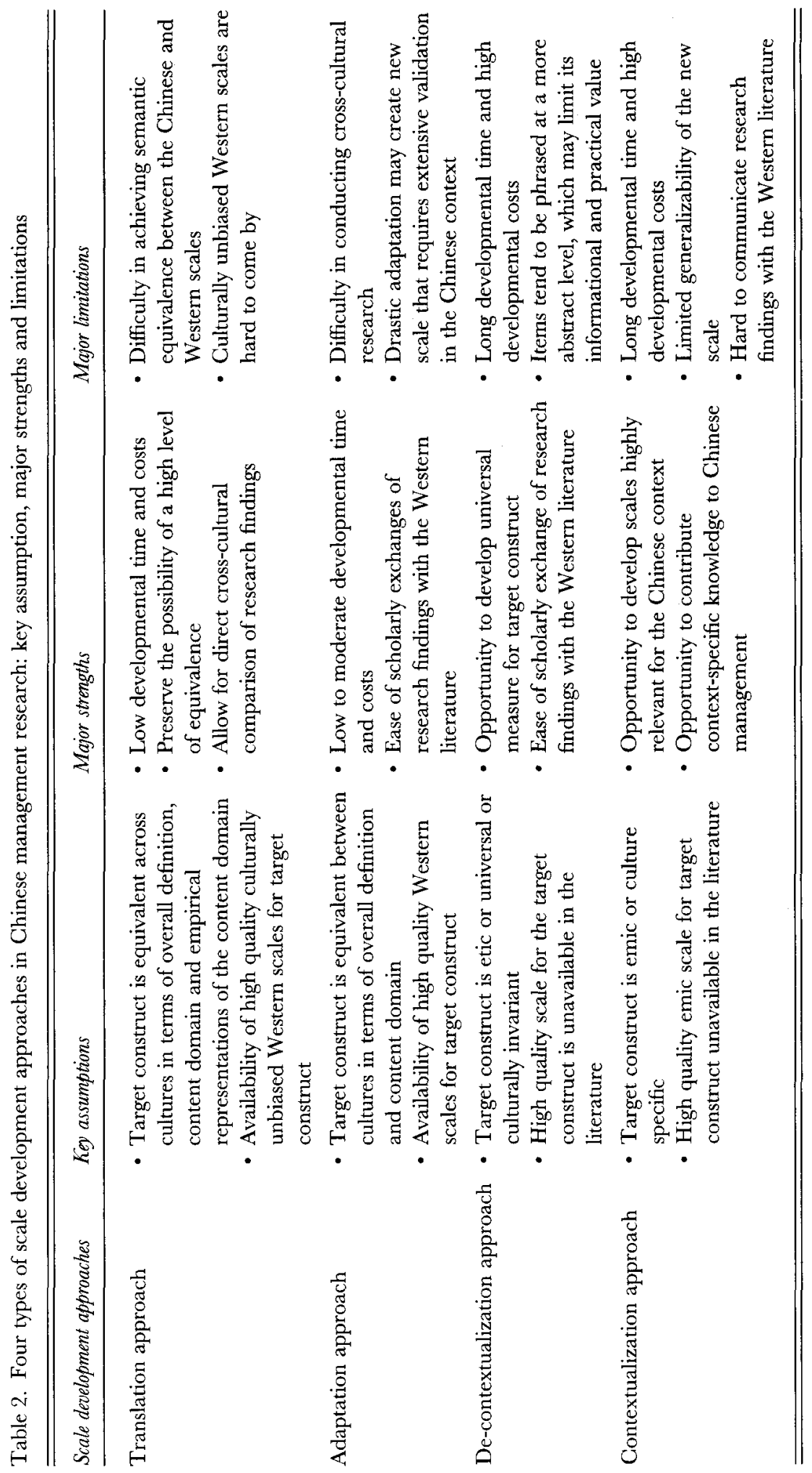


ensure that the source-language scale can be properly translated into the target language scale resulting such that the two versions of the scale are semantically equivalent. There are several translation techniques for accomplishing this goal (see Behling and Law, 2000, pp. 17-24), and the most widely used is the forwardand back-translation procedure (Brislin, 1980). As noted earlier, the translation approach also assumes the availability of a high quality and unbiased sourcelanguage scale. High quality here means that the scale has proven construct validity (Schwab, 1980), which implies that the scale has gone through a validation process and is judged to be valid for measuring its intended construct in the source language. The term 'unbiased' implies that the scale, along with its format and administration, is free of systematic errors across cultures (see Van de Vijver, 2003; Van de Vijver and Leung, 1997, for a discussion of the various types of biases in cross-cultural research).

Given the paucity of locally developed measures in China, coupled with the dominance of the Western research paradigm in management, most researchers use the direct translation approach to develop their scales. This approach saves development time and costs, and a properly translated scale allows researchers to compare their findings directly with those from Western settings. In addition, if the Western scale has already gone through extensive validation, its Chinese version can build on that research base, and thus does not need to go through the same costly validation procedure that a brand new scale would require. Indeed, translated Western scales have been widely used in Chinese management research. For example, in Wang et al. (2005), a study of leader-member exchange as a mediator of the relationship between transformational leadership and followers' performance and organizational citizenship behaviour in China, all four key variables (i.e., leader-member exchange, transformational leadership, performance and organizational citizenship behaviour) were measured with directly translated Western scales. Lam and Schaubroeck (2000) is another example. These authors reported a quasi-experimental study of the role of the locus of control in reactions to being promoted versus being passed over using a sample in Hong Kong. In the study the locus of control, organizational commitment, job involvement, job satisfaction and intention to quit were all measured with directly translated Western measures.

The translation approach is not without its limitations. One of its major drawbacks is that it is sometimes difficult to achieve semantic equivalence through literal translation because the cultural distance between the West and the East (China) is large. The other drawback is that even high quality, well established Western scales may contain unknown cultural biases, which automatically carry over to the Chinese scale through translation. To ensure that a measure is free of cultural bias, a de-centered approach is recommended, in which a culturally diverse perspective is taken in the conceptualization and design of a study (Werner and Campbell, 1970). However, few scales in social sciences have been constructed this way and 
existing Western measures were developed with no clear intent for cross-cultural application. The net result is that a translated scale may be biased in measuring the target construct in Chinese contexts. 'Transfer of validity (e.g., construct and predictive validity) from one cultural context to the other cannot be taken for granted but has to be demonstrated', especially when there is a large cultural distance between the two contexts (Van de Vijver and Hambleton, 1996, p. 95). These concerns are probably less serious for perceived objective characteristics (such as organizational structure, strategy and performance), but more so for psychological constructs (such as motivation, commitment and justice) whose meaning may be affected by social and cultural traditions.

\section{The Adaptation Approach}

To overcome the shortcomings of translation, the researcher may modify the source language scale to render it suitable for applications in Chinese contexts. Adapting a scale can include altering the wording of items, dropping inappropriate items and adding new items to the scale. While both translation and adaptation approaches require equivalence in the definition and content domain of the target construct, researchers who adapt measures generally do not assume that the empirical representations of the content domain are equivalent across cultures, or that the Western scale is free of cultural biases. This places the adaptation approach under an emic orientation.

Tsai (2001) studied the determinants and consequences of employees who displayed positive emotions among a sample of shoe store sales clerks in Taiwan. To measure displayed positive emotions, Tsai included four indicators from the Western literature (i.e., greeting, thanking, smiling and establishing eye contact) and two local indicators (i.e., asking customers to wait for a while and speaking in a rhythmic vocal tone). The two local indicators were identified based on interviews of 30 store supervisors and employees in Taiwan. Note that by applying the two local indicators to the four Western indicators, Tsai (2001) enriched the scale for the Chinese research context without altering the fundamental definition of the construct.

The adaptation approach enjoys the same benefits as the translation approach but to a lesser degree. The developmental costs for adaptation are somewhat higher than literal translation because adaptation requires that the researchers conduct some fieldwork (e.g., an expert panel or a focus group) to pinpoint the exact modifications that need to be made. If the researcher determines that a substantial amount of adaptation needs to be carried out, he or she will need to conduct a field study to demonstrate the psychometric qualities of the adapted scale before using it for substantive research. Moreover, since an adapted scale no longer contains an identical set of items as the source scale, it is more difficult to compare findings from adapted scales with those from the source scale. ${ }^{[2]}$ However, many 
Chinese researchers who adapt scales from the Western literature are primarily interested in a valid scale for local research, with no specific interest in direct cross-cultural comparisons. In such cases, the adapted scale and the source scale need not be strictly equivalent (Hambleton and Patsula, 1998). Based on our observation, the adaptation approach has been widely used in management research in China (e.g., Chen et al., 1998; Li and Atuahene-Gima, 2001), and for good reason. Given the large cultural distance between China and the West, simple translation of Western measures may not yield adequate instruments for Chinese contexts. With an awareness of the limitations of simple translation, researchers have begun to avoid the term 'translation' in favour of 'adaptation' to highlight the objective of ensuring conceptual equivalence in revising a source language scale for a different language and culture (Geisinger, 1994).

\section{The De-Gontextualization Approach}

Both the translation and adaptation approaches are designed to take advantage of established Western scales available in the literature. However, when a scale is not available, researchers must develop new scales. Approaches to new scale construction in Chinese contexts depend upon the researcher's assumptions about the etic versus emic nature of the target construct. The behavioural sciences are replete with examples of a construct once thought to be etic that later turned out to be emic, and vice versa (Smith and Bond, 2003). Nevertheless, in developing a new measure, the researcher should take a position on whether the target construct is perceived to be etic or emic in nature, given the current state of knowledge and the researcher's personal research orientation. An etic construct is one that the researcher believes to be universal or insensitive to cultural contexts, whereas an emic construct is one that the researcher believes to be culture-specific or unique to Chinese contexts. ${ }^{[3]}$ We consider the etic approach first.

With a de-contextualization approach, the researcher is striving to develop a measure for a new construct that transcends cultural groups. As the local context is presumed to be irrelevant to the construct, the researcher strives to de-contextualize the scale (i.e., context-specific factors are deliberately kept out of the scale construction process to avoid local cultural biases). The literature on cross-cultural psychology has a number of suggestions for designing a scale that is free of cultural biases (e.g., avoiding including local colloquialisms in a measure to increase its translatability) (please refer to Harkness et al., 2003).

A recent example of the de-contextualization approach is the Emotional Intelligence Scale recently developed by Wong and Law (2002) in Hong Kong. They first pointed out that there had been a lack of a psychometrically sound, but practical, emotional intelligence measure for leadership and management research. This justified their efforts to construct a new measure. Based on the conceptualization of emotional intelligence by Mayer and Salovey (1997), they 
constructed their scale deductively in Hong Kong and tested it with some six different samples. In the entire paper, they did not mention Chinese or Hong Kong cultural context at all! To them, the context of Hong Kong was theoretically irrelevant to their research effort. Their study could have taken place anywhere in the world with presumably similar findings.

Interestingly the de-contextualization approach does not seek to capitalize on the richness of Chinese contexts in scale development. Rather, the approach neutralizes the context (i.e., de-contextualizes it), removing it from the scale construction process. In doing so, it tends to phrase items at a more abstract level, which could reduce its informational and practical value for the local context. In the field of management, the de-contextualization approach undoubtedly dominates, though as Boyacigiller and Adler (1991) note, not necessarily because the scales were designed with de-contextualization in mind. Rather, most measures in the Western literature were created for Western contexts, and not much thought has gone into de-contextualizing them.

\section{The Gontextualization Approach}

The emic researcher takes an opposite view of the nature of the target construct and advocates a different scale development philosophy. Emic researchers believe that many constructs in behavioural sciences are embedded in local contexts and thus have culturally specific facets. The emic perspective can be viewed as part of a worldwide backlash against the 'unjustified' claims of universality of social science constructs developed in the West (Kim and Berry, 1993; Sinha, 1997). In order to correct the mistakes of imposed etics or pseudo-etics, emic researchers emphasize understanding of the local context from its own frame of reference - its ecological, historical, cultural and institutional contexts. Contextualization (or indigenization), which aims to maximize the appropriateness of psychological constructs and instruments to local cultures, often implies assembling different instruments for different cultural groups (Sinha, 1997).

In Chinese contexts, the contextualization movement in psychology began in the 1970s, led by Kuo-Shu Yang in Taiwan. In the last 30 years, Yang and his students have studied a number of constructs in social psychology from indigenous perspectives including: individual traditionality-modernity, social orientation, face, harmony, renqing (reciprocity in relationships) and yuan (predestined relationship) (Hwang, 2000; Yang, 1996, 1997). Psychologists in Hong Kong and mainland China led by Fanny Gheung have developed a contextual instrument called the Chinese Personality Assessment Inventory or CPAI (Cheung et al., 1996). The CPAI consists of personality scales that overlap with those covered by Western scales (i.e., dependability, social potency and individualism factors) but also include scales that are particularly relevant to Chinese culture (i.e., the interpersonal relatedness factor which includes harmony, face and renqing). Recent research 
conducted cross-culturally has shown that the interpersonal relatedness factor could not be subsumed under the big-five model of personality and is a new dimension of human personality (Cheung et al., 2001).

Are constructs and their facets uncovered in Chinese contexts using the contextualization approach but yet unknown to the West truly unique to China? The CPAI experience shows that what is presumed to be unique to China (such as harmony, renqing and face) could turn out to be very meaningful in the West. Indeed, it is difficult to 'prove' that a construct identified in China is unique to China for several reasons. ${ }^{[4]}$ First, these China-specific constructs have not been tested in Western cultures. Researchers simply 'assume' that they are not applicable in the West. Secondly, even if researchers have evidence to show that a translated version of the Chinese scale for the construct does not work in the West, they still cannot be sure if it is the 'construct' that is unique to China or the 'scale' that is unique to China. By using a de-contextualizing approach, researchers may be able to develop a version of the scale that works well in the West. These difficulties, together with non-random sampling and relatively small sample sizes in most empirical research, make a generalization of the conclusion that 'a construct is culturally emic' hazardous. Similarly, constructs that are presumed to be etic (universal) may not be truly etic until it can be shown to be valid or applicable in every cultural group. Thus, when a construct is labeled as emic (culturally unique or specific) or etic (universal), it is more of a researcher's working hypothesis than a fact, which can be validated or invalidated empirically in the future.

In the management area, researchers associated with the Hong Kong University of Science and Technology have developed several contextual (indigenous) measures including employment relationship, leadership style, loyalty to supervisor, organizational citizenship behaviour, and organizational culture (see Tsui, 2006 for a brief review). In each case, the scale development process began with presenting a construct and its current definition to Chinese respondents. Examples of behaviours or indicators that relate to the construct were generated using an open-ended questionnaire or a semi-structured interview. Content analysis of the behavioural samples resulted in a set of distinct categories, each of which was anchored by representative behavioural statements. These statements were then pilot tested in the field using factor analysis, which usually resulted in a set of measures that included dimensions similar to those found in the existing (Western) literature as well as some dimensions unique to the local context.

It has been observed that most of the influential academic research conducted in Chinese contexts has an indigenous nature (Tsui and Lau, 2002). These studies are all grounded in rich contextual information, not the simple replications that test existing Western models or findings in Chinese contexts. Indigenous measures developed from an emic perspective are uniquely suited to contribute to contextspecific knowledge about China as these measures are maximally relevant to the local context. However, the contextual approach is not without its limitations. 
First, it is costly in terms of time, effort and resources. Secondly, as contextual scales are not designed to be generalizable across cultures, they are not conducive to comparative research. Finally, there is always the danger that the indigenous constructs or measures may be too novel for mainstream researchers, which could create artificial barriers for their acceptance in international scholarly conversations (Whetten, 2002).

\section{THE FIVE PAPERS IN THE SPEGIAL ISSUE}

This special issue consists of five papers. The first four papers report the development or refinement of a scale or a set of measurement procedures for some theoretical constructs relevant to Chinese contexts. The fifth paper is a methodological piece in which the authors illustrated the use of structural equation modeling (SEM) to test or establish measurement equivalence or invariance for a pair of constructs or measures across 29 cultural groups. Based on the classification system described above, we would classify the first two papers (Delios, $\mathrm{Wu}$ and Zhong; Tsui, Wang and Xin) as representing the contextualization approach to the development of a new scale or measure. The third paper, by $\mathrm{Wu}$ and colleagues, and the fifth paper, by Tang et al. conform to the de-contextualization approach to new scale development and validation. The fourth paper by Xiao and Björkman uses the translation approach.

\section{Delios et al. - A Gase of the Contextualization Approach}

The first paper by Delios et al. develops a new conceptualization and classification scheme for ownership identities of China's listed firms. We classify this study as contextualization because the ownership structure and identities of Chinese listed firms are products of China's unique economic development experiences and institutional history. Many of the ownership identities that they describe have no counterparts outside China. Note, as we mentioned earlier, while our discussion emphasized scales, the approaches are broadly applicable to measure development. Delios and colleagues derive a classification scheme, not a scale.

Delios et al. point out that ownership structure has emerged as an important construct in the strategy literature in recent years. Like many variables in strategy research (e.g., competitive strategy or organizational structure), the operationalization of firms' ownership identities takes the form of a classification scheme, placing firms into mutually exclusive categories based on some attributes, and results in a nominal scale. In China, market and command economies are intertwined and hybrid organizational forms thrive. Delios et al. argue that the current conceptualization and classification scheme for ownership identities of Chinese listed firms, while sanctioned by Chinese governmental agencies, is inadequate for research because many of its categories (such as legal 
person shares) are highly ambiguous. This lack of precision introduces serious measurement error and renders the current scheme invalid for research. This may have contributed to the highly inconsistent findings regarding relationships between ownership identities and firm performance among Chinese listed firms.

The new perspective proposed by Delios et al. offers a more theory-aligned, precise and justifiable classification scheme for ownership identities among Chinese listed firms. The authors justify the validity of their new scheme through logic and illustrative cases, all of which are founded on their in-depth knowledge of the corporate setting in China. The new ownership classification scheme provides a timely tool for researchers interested in studying ownership structure among listed firms in China, and will help to unpack the inconsistent findings among ownership identities, strategy and performance in the literature.

\section{Tsui et al. - A Contextualization Approach}

In the paper entitled, 'Organizational culture in China: An Analysis of Culture Dimensions and Culture Types', Tsui et al. identify five common cultural values of state-owned enterprises, joint ventures/foreign wholly owned companies, and privately owned companies, and relate these five dimensions to the functions of internal integration and external adaptation values (Schein, 1992). In addressing the question of organizational culture varying among firms with different ownership structures and whether culture relates to firm performance or employee attitudes, as has been demonstrated in US firms, they conducted a series of three studies. They developed the cultural dimensions scale using both a qualitative and a quantitative approach. This two-phase design helps to ensure methodological rigour and capitalizes on the unique strengths of the two traditionally separate research orientations (Lee, 1999). They found that a highly integrative culture (similar to the profile of a 'strong' culture described in the Western literature), which promotes employee development and harmony as well as customer orientation, innovation and responsibility to the society as a whole, is most strongly associated with high ratings of firm performance by executives and positive employee attitudes as expressed by managers.

Chinese organizations are coping with the transition from a planned economy to a market oriented economy. The transition includes a de-emphasis on job security and more emphasis on productivity and performance-based rewards. The responsibilities of state-owned enterprises are shifting from the government or the state to the managing directors of enterprises (Liu, 2003). According to Liu (2003), this reformed environment in China has brought about changes in organizational culture as well as changes in the relationship between the individual and the organization. In this regard, this article is timely and reflects the changing Chinese management context. 
The Tsui et al. measure of Chinese organizational culture offers a good starting point, laying a solid foundation for further refinement by other scholars. The authors focused on five common dimensions of culture. By choosing a set of common dimensions (general across different ownership contexts), they compromised on the specific dimensions unique to each type of context (see their Table 3 for both the common and unique dimensions). Another area of refinement is in more clearly defining the content domain of each dimension. Further evidence on the convergent and discriminant validity of the measure is also desirable. For example, future studies could search for other measures to validate the dimensions of harmony, customer orientation, social responsibility, innovation and employee development. Tsui et al.'s measure is an excellent starting point for future studies on the organizational culture of Chinese companies.

\section{Wu et al. - A De-Contextualization Approach}

Social exchange theory is one of the most widely used theories when explaining employee behaviour in organizations (Cropanzano and Mitchell, 2005). After all, employment relationships are characterized by economic and social exchanges in which employees offer efforts, skills and contributions to their jobs in exchange for opportunities, rewards and benefits provided by employers. Wu et al.'s paper develops a new scale to operationalize the norm of reciprocity - a fundamental construct in social exchange theory. Building on Sahlins' (1972) conceptualization of norm of reciprocity as three distinct types (i.e., generalized, balanced and negative), the authors developed a multi-dimensional scale to assess the three reciprocity types in China. Despite the fact that the initial item pool for the scale was developed with input from Chinese researchers, managers and employees, the scale was tightly constructed, based on Sahlins' typology, which is presumed to be universal across cultures. The input from Chinese experts and respondents did not seem to alter either the overall definition or the specific content domain of the construct. Based on this logic, we classify this case as a de-contextualization approach to new scale development since the scale does not include any culturespecific items. We expect that the scale can be easily adapted to other cultural groups as a measure of reciprocity types, although it clearly is relevant for use in China.

$\mathrm{Wu}$ and his colleagues follow the standard procedure of new scale development (DeVellis, 2003; Hinkin, 1998). In study 1, based on Sahlins' typology, the authors developed an item pool to measure the three reciprocity types, conducted judge analysis to screen out bad items, and then performed factor analysis and item analysis to yield a valid and reliable scale. The factor structure of this new scale was then cross-validated in study 2 using an independent sample. In study 3 they further examined the nomological net of the new scale. The thorough procedures used in scale development provide good evidence that the new scale has construct 
validity for measuring what the researchers intended to measure - Sahlins' typology of reciprocity types. This scale should facilitate future research to advance our knowledge and understanding about the underlying processes as specified in the social exchange theory.

\section{Xiao and Björkman - An Example of the Translation Approach}

Xiao and Björkman's paper documents their effort to introduce a theory-based scale for high commitment work systems in China. Their scale was developed largely based on the definition and theory of high commitment work systems by Baron and Kreps (1999). In addition to the 12 high commitment work practices presented by Baron and Kreps (1999), they included two additional practices (i.e., performance appraisal and employee participation), which they judged as important in the Western literature, to complete their scale. Note that while the authors adjusted the original scale by adding two new practices to it, this decision was not derived on any specific consideration of the Chinese context - the two new practices came from Western contexts. They then applied the translation and back-translation procedure to create a Chinese version of the scale. The Chinese scale underwent field tests using two independent samples - one employee and one employer. Five items were then deleted due to low factor loadings on the primary factor, which resulted in a 9-item scale. We classify their scale development effort as a translation because all of the scale items (i.e., high commitment work practices) were taken from the existing literature. If they had added some items unique to Chinese contexts, it would become an adaptation approach.

Xiao and Björkman compared this scale to various measures of high commitment work practices in the literature to evaluate its content validity. They also presented some evidence on the convergent and discriminant validities of their 9-item scale with two Chinese samples, resulting in a preliminary scale of high commitment work practices. We would encourage research to further test its validity in the Chinese context and to use this scale to examine the impact of high commitment work practices on employee attitudes and behaviour as well as organizational performance in Chinese organizations.

\section{Tang et al. - Testing Measurement Equivalence/Invariance, Validation of an Etic Measure}

The fifth paper by Tang and his co-authors uses a data set of 29 samples from 29 countries/regions to illustrate the statistical procedures to assess the construct equivalence of a 'love of money' scale. By establishing measurement equivalence/ invariance (MEI) across these groups using structural equation modeling (SEM), the scale can be legitimately used in cross-cultural research. Such tests also involve a criterion measure of pay level satisfaction to establish functional equivalence 
across groups. After a series of elaborate procedures applied to both measures, the authors found only five of the 29 samples to have achieved the full measurement invariance.

While using SEM to test MEI across groups is a valuable tool for cross-cultural research, three cautionary notes are in order. First, different types of comparative research require different evidence in evaluating MEI. Full equivalence is not always needed (Vandenberg and Lance, 2000). A comparison of latent means between cultural groups requires support for configural invariance, metric invariance and intercept invariance. In contrast, testing functional equivalence between two variables across cultural groups requires only configural invariance and metric invariance.

Secondly, as Tang et al. mentioned in their article, researchers have not found a definitive set of analytic procedures and statistical tests for evaluating MEI using SEM. Experts in SEM have offered a complex set of guidelines involving different cut-off points for different goodness-of-fit indices for different forms of MEI under different testing conditions (e.g., equal vs. unequal sample sizes across groups) (see Chen, forthcoming, for details). The current procedures for testing MEI have also been found to be susceptible to contextual influences (Vandenberg, 2002). Crosscultural researchers should be aware of the limitations of the current procedures for testing MEI.

Thirdly, it may not be realistic to expect perfect invariance or congruence in global studies that involve many cultural groups. Only five of the 29 cultural groups in Tang et al.'s study survived the basic configural invariance test for both the love of money and pay level satisfaction scales. Leung and Bond (2004) argued that even if a perfect congruence structure exists for a measure among many cultural groups, the chance of identifying it is very small. This is because cross-cultural research involves many steps, each of which is vulnerable to random and systematic errors (Van de Vijver and Leung, 1997). For example, improper translation and uneven procedures followed in administering questionnaires introduce one source of error. People also tend to evaluate themselves relative to similar others in their own cultural group, which may constitute another source of error in cross-cultural comparison (Heine et al., 2002). Moreover, cultural differences produce a slightly different twist and slant to even the same concepts. All of these factors make perfect congruence or equivalence unrealistic in many field settings (Van de Vijver and Leung, 1997).

Given the above concerns, a strict and rigid application of MEI in cross-cultural research could err on overkill. However, let us not throw out the baby with the bath water. We concur with Tang et al. that a more sensible approach seems warranted. They suggest that when support for strict MEI is rejected, researchers could consider applying a more liberal standard and explore alternative solutions (e.g., identifying and eliminating the misfit items, allowing for partial invariance). Dropping data that do not conform to MEI should be a last resort. 


\section{CONGLUSION}

With the rise of the Chinese economy and the need for locally relevant management knowledge, more and more survey measures are imported or developed for management research in Chinese contexts. In this article, we have delineated four distinct approaches to scale development in international management research with the specific consideration of China. Each approach has its unique strengths and weaknesses. These four scale development approaches are best viewed as four specialized tools in a researcher's toolbox. Just like a master chef who needs to apply different knives when preparing different foods, a good empirical researcher needs to select the right tools to measure variables. The five papers in our special issue provide solid examples of the scale development approaches we identified, beyond the commonly used approach of translating existing Western scales found in most studies. They are part of a broader effort to develop valid instruments for Chinese (and other non-Western) organizations and management research. We hope to see more of such effort in the future. As Li and Tsui (2000) observed, the survey has been the most commonly used method for data collection in Chinese management research with hundreds of Chinese-language measures being used in research and published in the mainstream management journals. To maintain and enhance the quality of Chinese management research, future research should systematically review this body of literature to find out how the four different approaches to scale development have been used in published research and to identify the challenges and best practices associated with each approach (c.f. Clark and Watson, 1995). With improved measures over time, the development of management knowledge in Chinese contexts will accelerate in the years to come.

\section{NOTES}

We would like to thank Kenneth Law, Jian Liang and Anne Tsui for their comments on an earlier version of this paper and Mingjian Zhou for his assistance in reviewing the literature on measures in Chinese management research.

[1] Our discussion emphasizes scale development, but the processes we describe are broadly appropriate for many non-verbally mediated measures as well. The approaches we discuss are fundamentally about deriving measures that reliably and validly capture underlying constructs, and the resulting measures can range from psychological instruments to objective indices or categories.

[2] In cross-cultural research, it may be reasonable to ignore culturally specific items and focus on universal items only - especially when the items are considered reflective indicators of the construct.

[3] Constructs that are complex in nature may be partly etic and partly emic. That is, they include both etic and emic facets. If researchers are interested in etic facets only, they should of course take a de-contextualization approach. If researchers are interested in uncovering emic as well as etic facets in a specific cultural context, they should take a contextualization approach (e.g., Cheung et al., 1996, 2001; Farh et al., 1997, 2004). After all facets of the construct have been identified, they can then separate etic ones from emic ones by comparing their findings with those from other cultural groups using a similar approach.

[4] We would like to thank Kenneth Law for bringing these reasons to our attention. 


\section{REFERENGES}

Baron, J. N., and Kreps, E. D. (1999). Strategic Human Resources. New York: John Wiley and Sons.

Behling, O., and Law, K. S. (2000). Translating Questionnaires and Other Research Instruments: Problems and Solutions. Thousand Oaks, Calif.: Sage Publications.

Boyacigiller, N. A., and Adler, N. J. (1991). 'The parochial dinosaur: Organizational science in a global context'. Academy of Management Reviewe, 16, 262-90.

Brislin, R. W. (1980). 'Translation and content analysis of oral and written material'. Handbook of Cross-Cultural Psychology, 2, 339-444.

Chen, F. F. (forthcoming). 'Sensitivity of goodness of fit indices to lack of measurement invariance'. Structural Equation Modeling.

Chen, X.-P., Hui, C., and Sego, D. J. (1998). 'The role of organizational citizenship behavior in turnover: Conceptualization and preliminary tests of key hypotheses'. Foumal of Applied Psychology, 83, 922-31.

Cheung, F. M., Leung, K., Fan, R. M., Song, W. Z., Zhang, J. X., and Zhang, J. P. (1996). 'Development of the Chinese Personality Assessment Inventory'. Foumal of Cross-Cultural Psychology, 27, 181-99.

Cheung, F. M., Leung, K., Zhang, J. X., Sun, H. F., Gan, Y. Q., Song, W. Z., and Xie, D. (2001). 'Indigenous Chinese personality construct: Is the Five-factor model complete?'. Journal of CrossCultural Psychology, 32, 407-33.

Clark, L. A., and Watson, D. (1995). 'Constructing validity: Basic issues in objective scale development'. Psychological Assessment, 7, 309-19.

Cropanzano, R., and Mitchell, M. S. (2005). 'Social exchange theory: An interdisciplinary review'. Joumal of Management, 31, 874-900.

DeVellis, R. F. (2003). Scale Development: Theory and Application, 2nd edn. Thousand Oaks, Calif:: SAGE Publications.

Farh, J. L., Earley, P. C., and Lin, S. C. (1997). 'Impetus for action: A cultural analysis of justice and organizational citizenship behavior in Chinese society'. Administrative Science Quarterly, 42, 42144.

Farh, J. L., Zhong, C. B., and Organ, D. W. (2004). 'Organizational citizenship behavior in the People's Republic of China'. Organization Science, 15, 241-53.

Geisinger, K. F. (1994). 'Cross-cultural normative assessment: Translation and adaptation issues influencing the normative interpretation of assessment instruments'. Psychological Assessment, 6, 304-12.

Hambleton, R. K., and Patsula, L. (1998). 'Adapting tests for use in multiple languages and cultures'. Social Indicators Research, 45, 153-71.

Harkness, J. A., Van de Vijver, F. J., and Mohler, P. P. (2003). Cross-Cultural Survey Methods. Hoboken, N.J.: John Wiley \& Sons.

Heine, S. J., Lehman, D. R., Peng, K., and Greenholtz, J. (2002). 'What's wrong with cross-cultural comparisons of subjective Likert scales?: The reference-group effect'. Joumal Personality and Social Psychology, 82, 903-18.

Hinkin, T. K. (1998). 'A brief tutorial on the development of measures for use in survey questionnaires'. Organizational Research Methods, 1, 104-21.

Hwang, K. K. (2000). 'Chinese relationalism: Theoretical construction and methodological considerations'. Foumal for the Theory of Social Behavior, 30, 155-78.

Kim, U., and Berry, J. W. (1993). Indigenous Psychologies: Research and Experience in Cultural Context. Newbury Park, Calif:: Sage.

Korman, A. K. (1974). 'Contingency approaches to leadership'. In Hunt, J. G., and Larson, L. L. (Eds), Contingency Approaches to Leadership. Carbondale, Ill.: Southern Illinois University Press.

Lam, S. S. K., and Schaubroeck, J. (2000). 'The role of locus of control in reactions to being promoted and to being passed over: A quasi experiment'. Academy of Management Joumal, 43, 66-78.

Lee, T. W. (1999). Using Qualitative Methods in Organizational Research. Thousand Oaks, Calif:: Sage.

Leung, K., and Bond, M. H. (2004). 'Social axioms: A model for social beliefs in multicultural perspective'. Advances in Experimental Social Psychology, 36, 119-97.

$\mathrm{Li}, \mathrm{H}$., and Atuahene-Gima, K. (2001). 'Product innovation strategy and the performance of new technology ventures in China'. Academy of Management foumal, 44, 1123-34.

Li, J. T., and Tsui, A. S. (2000). Management and Organizations in the Chinese Context. London: McMillan Press. 
Liu, S. (2003). 'Cultures within culture: Unity and diversity of two generations of employees in state-owned enterprises'. Human Relations, 56, 387-417.

Mayer, J. D., and Salovey, P. (1997). 'What is emotional in telligence?'. In Salovey, P., and Sluyter, D. (Eds), Emotional Development and Emotional Intelligence: Educational Implications. New York: Basic Books, (pp. 3-34).

Sahlins, M. (1972). Stone Age Economics. New York: Aldine de Gruyter.

Schein, E. H. (1992). Organizational Culture and Leadership, 2nd edn. San Francisco, Calif.: Jossey-Bass.

Schwab, D. P. (1980). 'Construct validity in organizational behavior'. Research in Organizational Behavior, 2, 3-43.

Sinha, D. (1997). 'Indigenizing psychology'. In Berry, J. W., Poortinga, Y. H., and Pandey, J. (Eds), Handbook of Cross-Cultural Psychology: Theory and Method. Boston, Mass.: Allyn \& Bacon, (pp. 129-69).

Smith, P. B., and Bond, M. H. (2003). 'Honoring culture scientifically when doing social psychology'. In Hogg, M. A., and Cooper, J. (Eds), Sage Handbook of Social Psychology. London: Sage, (pp. 43-61).

Tsai, W. C. (2001). 'Determinants and consequences of employee displayed positive emotions'. Joumal of Management, 27, 497-512.

Tsui, A. S. (2006). 'Contextualization in Chinese management research'. Management and Organization Review, 2, 1-13.

Tsui, A. S., and Lau, C. M. (2002). The Management of Enterprise in the People's Republic of China. Boston, Mass.: Kluwer Academic Press.

Van de Vijver, F. J. R. (2003). 'Bias and equivalence: Cross-cultural perspectives'. In Harkness, J. A., Van de Vijver, F. J., and Mohler, P. P. (Eds), Cross-Cultural Survey Methods. Hoboken, N.J.: John Wiley \& Sons, (pp. 143-56).

Van de Vijver, F. J. R., and Hambleton, R. K. (1996). 'Translating tests: Some practical guidelines'. European Psychologist, 1, 8999.

Van de Vijver, F. J. R., and Leung, K. (1997). Methods and Data Analysis for Cross-Cultural Research. Thousand Oaks, Calif.: Sage Publications.

Vandenberg, R. J. (2002). 'Toward a further understanding of and improvement in measurement invariance methods and procedures'. Organizational Research Methods, 5, 139-58.

Vandenberg, R. J., and Lance, C. E. (2000). 'A review and synthesis of the measurement invariance literature: Suggestions, practices, and recommendations for organizational research'. Organizational Research Methods, 2, 4-69.

Wang, H., Law, K. S., Hackett, R. D., Wang, D., and Chen, Z. X. (2005). 'Leader-member exchange as a mediator of the relationship between transformational leadership and followers' performance and organizational citizenship behavior'. Academy of Management fournal, 48, 420-32.

Werner, O., and Campbell, D. (1970). 'Translating, working through interpreters, and the problem of decentering'. In Norall, R., and Cohen, R. (Eds), A Handbook of Method in Cultural Anthropology. New York: Natural History Press, (pp. 398-429).

Whetten, D. A. (2002). 'Constructing cross-context scholarly conversations'. In Tsui, A. S., and Lau, G. M. (Eds), The Management of Enterprises in the People's Republic of China. Boston, Mass.: Kluwer Academic Publishers, (pp. 29-47).

Wong, C. S., and Law, K. S. (2002). 'The effects of leader and follower emotional intelligence on performance and attitude: An exploratory study'. The Leadership Quarterly, 13, 243-74.

Yang, K. S. (1996). 'Psychological transformation of the Chinese people as a result of societal modernization'. In Bond, M. H. (Ed.), The Handbook of Chinese Psychology. Hong Kong: Oxford University Press, (pp. 479-98).

Yang, K. S. (1997). 'Theories and research in Chinese personality: An indigenous approach'. In Rao, H. S. R., and Sinha, D. (Eds), Asian Perspectives on Psychology. Thousand Oaks, Calif.: Sage, (pp. 236-62). 
Jiing-Lih Farh (mnlfarh@ust.hk) is Chair Professor at Hong Kong University of Science and Technology. His research interests primarily focus on using an indigenous approach to investigating organizational behaviour in Chinese contexts (such as guanxi, paternalistic leadership and organizational citizenship behaviour). He currently serves as Senior Editor for the Management and Organization Review. He serves or has served on the editorial boards of over a dozen journals including the Academy of Management Foumal, Journal of Management, Personnel Psychology, Journal of International Business Studies, and Human Relations.

Albert A. Cannella Jr. (albert.cannella@asu.edu) is Hahnco Companies Professor of Strategic Management and Chair of the Management Department at Arizona State University. He received his Ph.D. from Columbia University in 1991. He serves on the editorial review boards of the Academy of Management Journal, Joumal of Management, and Administrative Science Quarterly. His research interests focus on executives, entrepreneurship and competitive dynamics.

Cynthia Lee (c.lee@neu.edu) is the Walsh Professor at Northeastern University, Boston, Mass. Her research interests include employment relationships, performance management and organizational change especially in Chinese contexts. She currently serves as Associate Editor for Applied Psychology: An International Review. She is the editorial board member of the Joumal of Applied Psychology and Fournal of Organizational Behavior. 